\title{
Tokenization of Assets Using Blockchain Technology
}

\author{
Rohit Dubey ${ }^{1}$ | Prof. Ajay Kaushik ${ }^{2}$

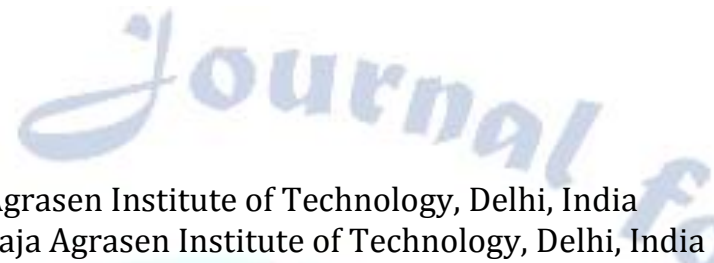 \\ ${ }^{1}$ B.Tech Scholar, Department of IT, Maharaja Agrasen Institute of Technology, Delhi, India
${ }^{2}$ Assistant Professor, Department of IT, Maharaja Agrasen Institute of Technology, Delhi, India
}

To Cite this Article

Rohit Dubey and Prof. Ajay Kaushik, "Tokenization of Assets Using Blockchain Technology", International Journal for Modern Trends in Science and Technology, 6(12): 202-207, 2020.

\section{Article Info}

Received on 10-November-2020, Revised on 30-November-2020, Accepted on 04-December-2020, Published on 09-December-2020.

\section{ABSTRACT}

In the ongoing occasions, Blockchain Technology (BCT) has additionally gotten impressive consideration outside the monetary area. The idea of Blockchain is much past than Bitcoin and Cryptocurrencies. Investigating the odds inside the Blockchain universe, various uses of BCT in Tokenization of assets are frequently actualized. During this paper, we will join these two viewpoints on BCT in Tokenization of assets to sum up the present status of the transactions and the future scope of such transactions in the future. This paper means to examine the part of Blockchain in maintainable transactions of actual money liquified into tokens and distributed through a blockchain network.

KEYWORDS: Blockchain Technology, Tokenization of Assets, Smart Contracts.

\section{INTRODUCTION}

Tokenization of assets refers to liquidating any real world assets into tokens, now this idea may not sound as exciting but the fact that we have the technology to convert any assets into tokens is amazing.

This will not only boost the market economy by increasing the flow of money but also has other benefits such as faster and cheaper transactions, more transparency (as blockchain is a public distributed ledger, so chances of a fraud/fault occurring fall drastically and lastly tokenization could open up Investment in assets to a much wider audience thanks to reduced minimum investment amounts and periods.

Traditionally, real world assets like cars, houses, jewellery etc., are liquidated to actual money. The whole process can be considered as flawed when compared to tokenization using cryptocurrency.Considering traditional methods one usually has a middle-person who makes any deal possible, now this person has commission. This is one such example of the flaws of this system.A blockchain network will help develop decentralized systems, where only the parties concerned will deal with each other.

\section{ASSET TOKENIZATION PROCESS}

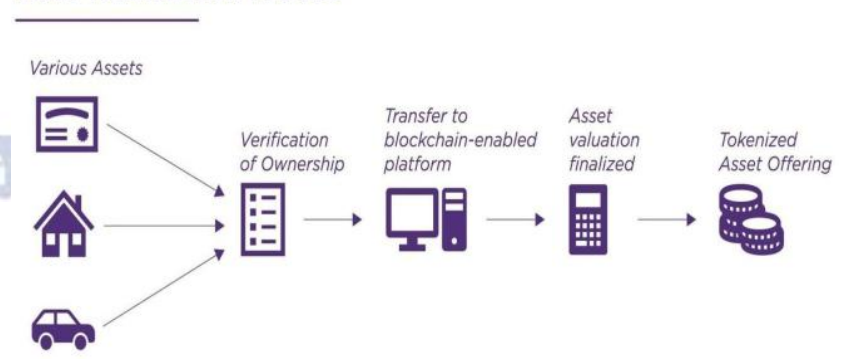

\section{Decentralization}

Decentralization is the exchange of power and duty regarding public capacities from the focal government to subordinate or semi autonomous government associations and additionally the private area. Decentralized account, otherwise 
called $\mathrm{DeFi}$, is a quickly developing area of the cryptographic money industry. While digital money coins make a decentralized store of significant worth separate from any administration sponsored fiat cash, DeFi makes decentralized monetary instruments separate from conventional concentrated organizations.

\section{BLOCKCHAIN TECHNOLOGY}

The Blockchain, a decentralized and scrambled advanced record, was recognized together of the most elevated 10 arising innovations inside the World Economic Forum in 2016. Blockchain might be such a game plan. It is regularly seen as a decentralized data set during which data are frequently put away. This information base is conveyed over all taking an interest hubs, which all concur on a specific arrangement of rules, with respect to the permitted conduct inside the organization and to the structure of the information put away. Blockchain is implied all together that all put away substances are permanent. This empowers all hubs to have admittance to the record as a permanent wellspring of information.

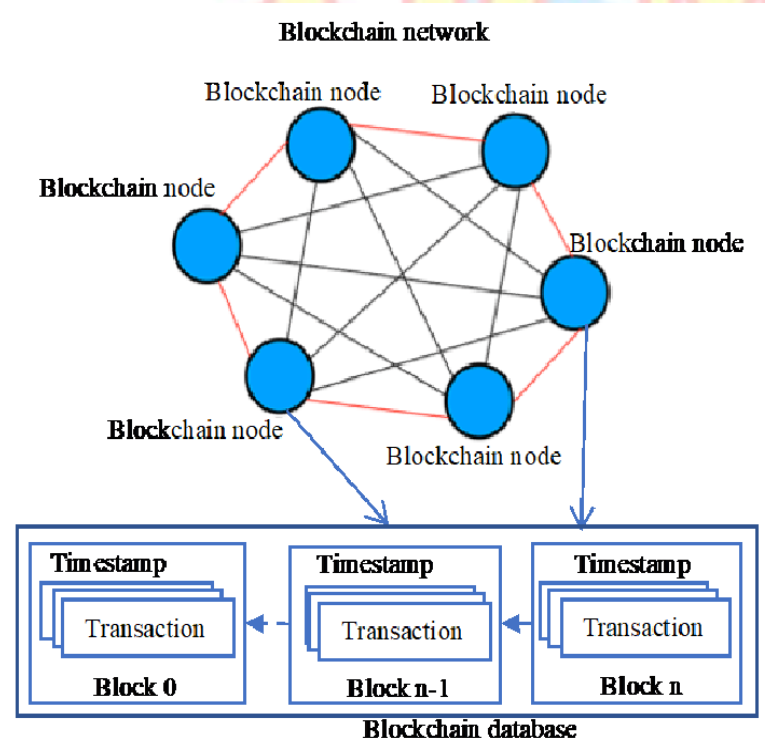

Blockchain as the name proposes is comprised of two words block and chain, as it were it is really a chain of blocks where the blocks are comprised of data. The data is the information of exchanges, which contains the hash key and exchange subtleties. Blockchain is ordinarily an appropriated shared open source record where every exchange is recorded with computerized marks. Consider it circulated information base put away in various PCs where regardless of whether one PC bombs the information base would even now be protected as its put away on various PCs.
Blockchain works when exchanges happen. The straightforward cycle is that first exchanges happen then they get confirmed and the block gets added to the chain. Each exchange happening in the Blockchain is checked and approved by miners or nodes in the organization. Verification of work is the agreement calculation that is utilized in Blockchain to check the exchanges in the Blockchain. [2] Now the check of exchanges resembles a riddle where the miner tackles the POW issue whose answer is the hash. Miners utilize their computational capacity to unravel this and get compensated in digital forms of money and so on.At the point when exchange gets checked the block gets added to the chain. Blockchain is a convention, its open source and anybody can see it, the exchanges stay mysterious or pseudo anonymous. Blocks as a rule contain about a normal of 500 transactions.Blocks are comprised of block header and exchange information.

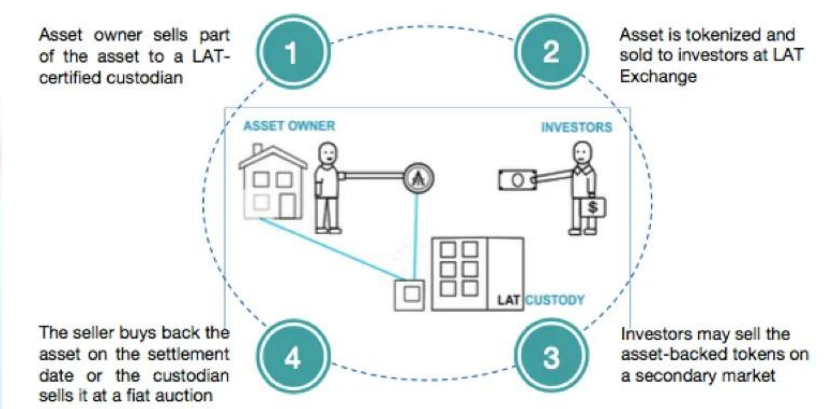

A computerized cash as well as a convention of agreement was made by Nakamoto, the maker of Bitcoin, giving trust even without the focal go-between and and dealing on a peer-to-peer network. The convention is predicated on three essential columns to gracefully establish this trust inside the framework: decentralization, agreement and cryptography.[1]

\section{SMART CONTRACTS}

Smart Contracts are self-executing contracts containing the terms and conditions of a game plan among peers. The terms and conditions of the plan are formed into code. The smart contracts are executed on the Ethereum Blockchain's decentralized network. The courses of action empower the exchanging of money, offers, property, or any asset. There are two by and large used programming languages for forming Ethereum Smart agreements - Solidity and Serpent. Heartiness is a raised level programming language used for realizing splendid agreements on the Ethereum Blockchain stage. It empowers Blockchain designers to check the program at run-time rather than gather time[3]. 
The sheer number of go betweens and midway layers related with the execution of an ordinary contracts moves back the cycle, habitually taking days or even weeks.

Smart Contracts are very efficient as they take literally minutes, as they are mechanized and programmable, running on a PC under predefined conditions. There are no external entities included. Insurance and security are stressed with standard contracts. With so many widely appealing gatherings included, security can be sabotaged at any stage all the while. Security is kept up through cryptography, public key, and private keys while using Smart Contracts. Kept up in a decentralized system, the data is practically hard to change. Smart Contracts are deliberately stamped using private keys and should be decoded by the public key shared by the social events being referred to.

Terms and conditions of a smart contract are pre determined.At the point when a condition is met, settlement happens therefore and is recorded. If any settlement is related to regular agreements, it's a manual cycle including support work measures. Usually, straightforwardness is coordinated by the social affairs being referred to, periphery substances, and center individuals. It's a flawed structure. Brilliant agreements, nevertheless, are $100 \%$ direct, open online $24 * 7 * 365$. Anyone can overview, survey, and support the reported trades. Archiving is problematic with customary agreements, as they are paper-based and cared for detached. Following trades is stumbling. Trades in Smart agreements may be followed straightforwardly from the motivation behind origin, and recording happens subsequently, making a totally open history. [5]

Traditional contracts are exorbitant when appeared differently in relation to smart contracts fundamentally in light of the fact that all of those go betweens must be paid. Smart contracts have off limits betweens, and the fundamental trade charges come from the essential system of the Blockchain network running the Smart contract.[10]

\section{LITERATURE SURVEY}

The tokenization of resources alludes to the cycle of giving a blockchain token (explicitly, a security token) that carefully speaks to a genuine tradable resource-from numerous points of view like the customary cycle of securitization, with an advanced twist.These security tokens would then be able to be exchanged on an auxiliary market. These security tokens are made through a kind of Initial Coin Offering (ICO)/Security Token Offering (STO). Additionally, these tokens are produced and overseen through smart contracts which makes these tokens secure against control and since blockchain is a conveyed record subsequently no exchange will continue until each gathering's records coordinate. [7]

Now the question arises : Why convert to tokens instead of actual money.Here are some advantages: A new "token economy" offers the potential for a more effective and more attractive monetary world by significantly decreasing the grating engaged with the creation, purchasing and selling of resources.

There are four key focal points that tokenization accommodates the two speculators and dealers:

- More noteworthy Liquidity:By tokenizing resources, particularly private protections or regularly illiquid resources, for example, fine art,property, adornments, and so on these tokens can be then exchanged on an auxiliary market of the backer's decision. This admittance to a more extensive base of dealers expands the liquidity.

- Quicker and Cheaper Transactions:Since the exchange of tokens is finished with keen contracts/software calculations incorporated into a blockchain with trigger activities dependent on pre-characterized boundaries certain pieces of the trade cycle are mechanized. This mechanization can decrease the regulatory weight engaged with purchasing open and selling, with less mediators required, prompting quicker arrangement execution, yet additionally lower exchange charges.

- More Transparency : A security token is equipped for having the token-holder's privileges and lawful obligations implanted straightforwardly onto the token, alongside an unchanging record of possession. These attributes guarantee to add straightforwardness to exchanges, permitting you to know with whom you are managing, what your and their privileges are, and who has recently possessed this token.

- More Accessible:Significantly, tokenization could open up Investment in resources for a lot more extensive crowd because of 
decreased least speculation sums and periods. Tokens are exceptionally distinguishable, which means speculators can buy tokens that speak to tiny rates of the hidden resources. On the off chance that each request is less expensive and simpler to measure, it will open the route for a huge decrease of least venture sums. Besides, the higher liquidity of security tokens could likewise decrease least speculation periods since financial specialists can trade their tokens on the optional business sectors, which are hypothetically worldwide and every minute of every day (subject as far as possible)

Among the game plan of Blockchain types of progress that are by and by set up and applied in various zones of business, coming up next are three of the most popular ones. Each Blockchain has unquestionable explicit attributes, thus intriguing application alternatives and central focuses. For this situation study, we will consider the characteristics of Hyperledger Fabric, Ethereum and Corda Blockchain options in contrast to diagram which one would be more significant to deftly chain the board applications.

\section{Example use cases}

\section{Case 1 - TheArtToken (TAT)}

Prints of unique works of art by celebrated specialists are regularly sold by the proprietors of the craftsmanship pieces. We should accept there are around 1,000 prints of some canvas.

These prints could be tokenized by having a specific organization hold responsibility for prints. The organization would then be able to offer general society to reclaim the tokens for a solitary duplicate.

When recovered, the actual conveyance can be made to the given location.

In the event that the estimation of the tokens reclaimed, doesn't coordinate a specific edge, at that point the symbolic holder can have the responsibility for part of the workmanship print as per its evaluated esteem.

TheArtToken (TAT), as an example, offers tokens that talk to part responsibility for and Post War Art. The first actual works of art are put away in a distribution center made sure about by the Swiss government.
Such tokenization of craftsmanship makes it less difficult for purchasers and outsider business sectors to execute in portions of works of art.[8]

\section{Case 2 - JP Morgan}

Despite the fact that the market managing the trading of products has massively moved to paperless exchanges, the overhead for these exchanges is immense.

Be that as it may, in what capacity can actual resources be tokenized? How about we take the case of gold here to comprehend the idea better. Suppose the gold in a vault is claimed by ABC, and the vault itself is possessed by XYZ.

$A B C$ could then offer responsibility for part of the gold to people in general as a computerized token. They could keep up the possession vault of the gold with an agreement with XYZ.

For each token sold, the responsibility for much measure of gold is moved to XYZ. A symbolic holder would then be able to recover the gold by demonstrating their proprietorship with the assistance of a computerized mark or declaration. JP Morgan, the American bank and monetary administrations monster, has reported that they would introduce gold bars as a tokenized resource on Quorum, their blockchain network.

\section{CASE 3 : CargoX}

The coordinations business depends on the strategy for giving verification of possession i.e., the Bill of Lading. This strategy causes various issues, for example, delay in conveyance and recovering of products on the off chance that the bill of replenishing is lost or lost, and so on

CargoX has concocted the arrangement of tokenization to evade the issues that are brought about by the customary strategy that utilizes the Bill of Lading (B/L). They are making an open framework dependent on Ethereum called Smart B/L.

Their Smart B/L framework is required to work comparably to the symbolic framework. At the cause, the transporter will make a Smart B/L with the assistance of their application and send it to the exporter.

When the shipper moves cash to the exporter, the exporter moves the responsibility for Smart B/L tokens to the merchant. At the less than desirable end, the shipper can guarantee the responsibility 
for products by introducing the Smart B/L token to the transporter.

\section{V.METHODOLOGY}

The following procedure was followed in fabricating this project:

\section{The ERC-20 Smart Contract}

The first smart contract is the Token. We don't invent it ourselves, we take the ERC20 Smart Contract from OpenZeppelin. In this version open-zeppelin v3, with Solidity 0.6 smart contracts:

Let's think about a possible work-scenario. We will create a Token which lets you redeem a coffee at your favorite Coffee Store: StarDucks Coffee from Duckburg. It will look slightly different from other ERC20 tokens, since a coffee is hardly divisible, but still transferable and thus we create our token.

\section{Add in a Migration for the Smart Contract}

A file named 2_deploy_contracts.js was added to the migrations part.

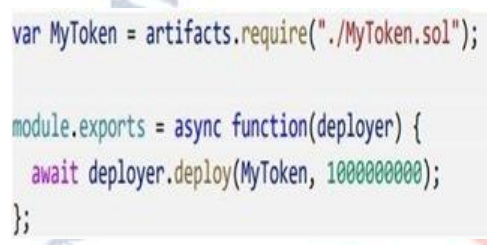

\section{Adjustment of truffle-config.js file}

The truffle-config file was adjusted to ensure that the default development network uses the right host port.

\section{Testing the ERC-20 Smart Contract}

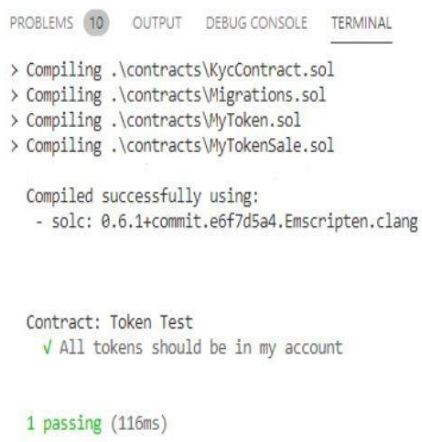

\section{Adding the actual Crowdsale Contracts}

Here two steps were performed:

1. We adapt the old Crowdsale Smart Contract from Open-Zeppelin to be Solidity 0.6 compliant.

2. We write our own Crowdsale on Top of it.
With OpenZeppelin moving toward Solidity 0.6 the Crowdsale contracts were taken out. A few people are slanted to add a "mintToken" usefulness or something to that effect to the Token Smart Contract itself, yet that would be an awful plan. We should add a different Crowdsale Contract that handles token circulation.

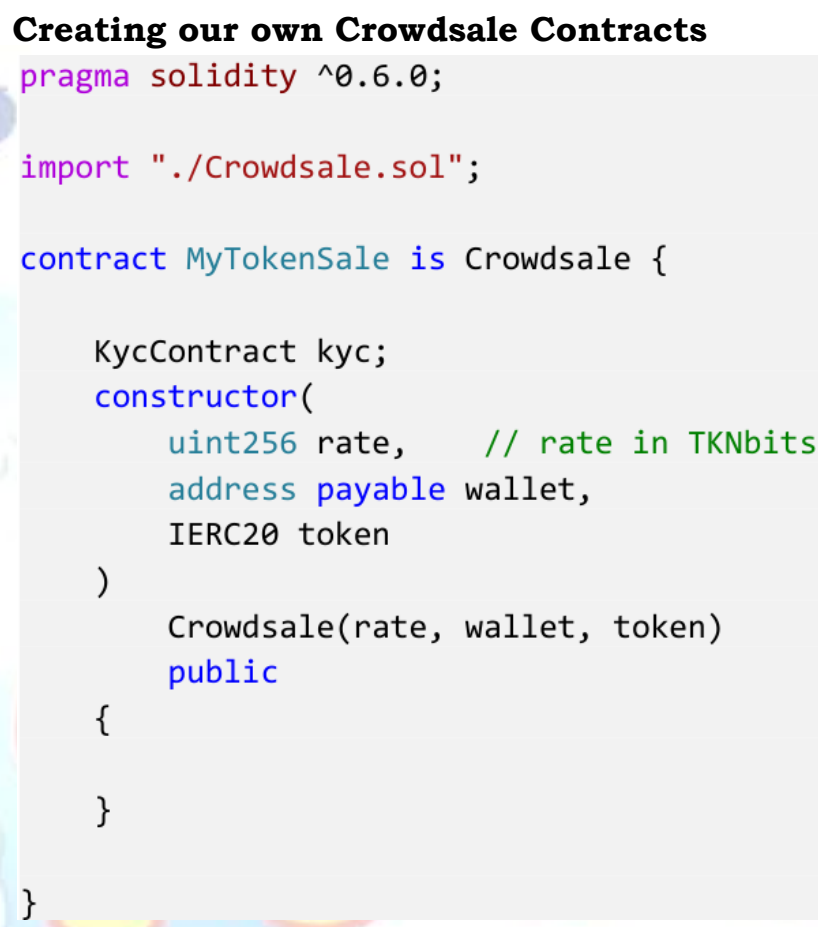

Adding KYC Mockups to verify the genuineness of a user

Not everyone will be able to purchase tokens a user must be validated through a Know Your Customer(KYC).The know your client or know your customer rules in monetary administrations necessitates that experts put forth an attempt to confirm the character, reasonableness, and dangers engaged with keeping up a business relationship. The methodology fit inside the more extensive extent of a bank's Anti-Money Laundering strategy.

The methodology fit inside the more extensive extent of a bank's Anti-Money Laundering strategy.

pragma solidity ^0.6.0;

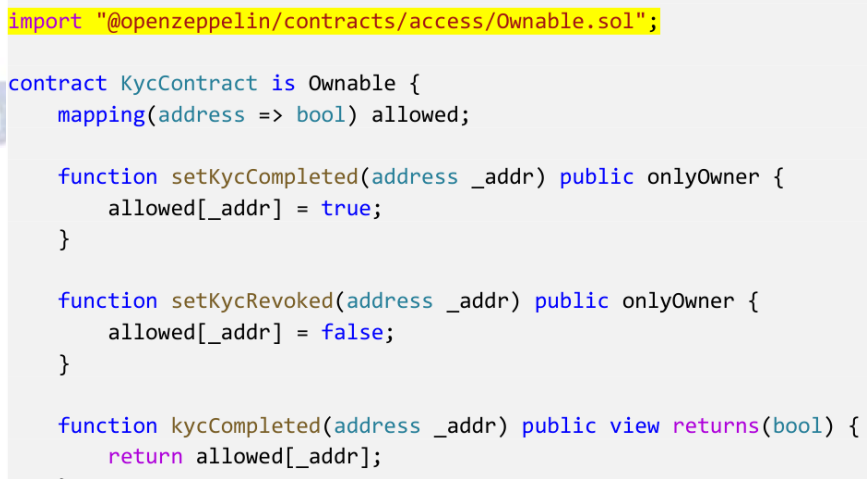




\section{The Frontend}

The front-end of this project is fabricated using the Javascript Library ReactJS

\section{ReactJS}

ReactJS is an open-source, front end, JavaScript library for building UIs or UI segments. It is kept up by Facebook and a network of individual designers and organizations. ReactJS can be utilized as a base in the advancement of single-page or versatile applications.

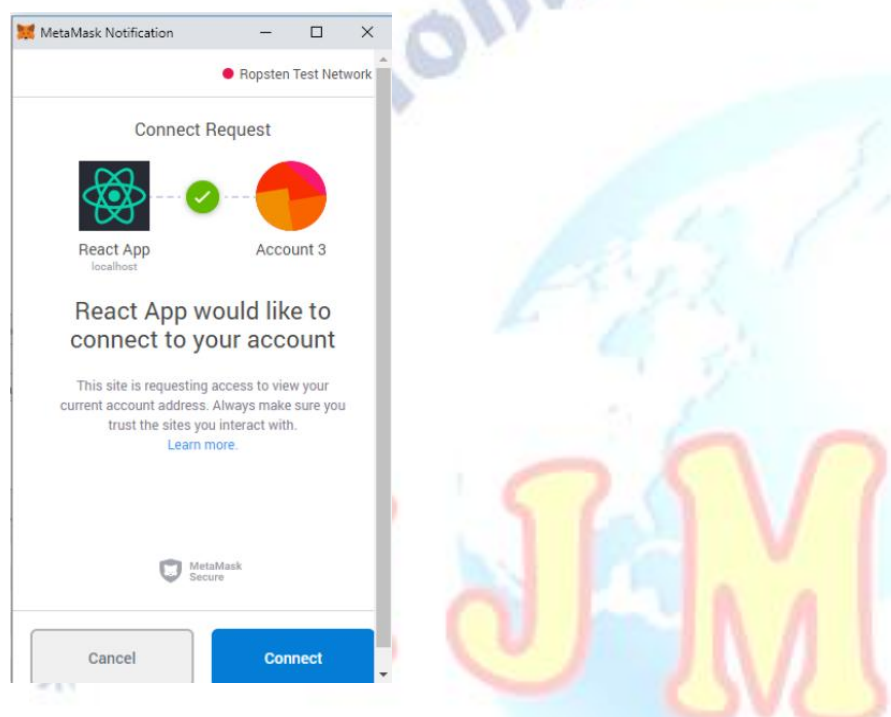

\section{VI.RESULTS}

The system fabricated can be used to for tokenization of assets of any kind though for this project a coffee shop blockchain token system was created

\section{Capuccino Token for StarDucks}

Enable your account

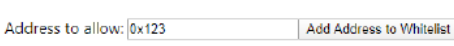

Buy Cappucino-Tokens

Send Ether to this address: 0x89416e57C05DF790F80E46d32cBe719F7DDF8aCd

You have: 11

Buy more tokens

There will be discrete dashboards for clients at each stage showing the data important to them and the administrator dashboard will approach all the data about tokens and some authority over who can see the data just as expansion and updation of clients just as tokens.

Also users must complete the KYC process in order to verify their genuineness so they may start publishing items for sale and so will be the case when users have to buy tokens/purchase any items.

\section{VII.ACKNOWLEDGMENT}

I thank my Mentor Prof. Ajay Kaushik for analyzing the data and for advising on all aspects related to this paper, along with other concepts too. Later for validating the experimental result and reviewed the paper.

\section{VIII.CONCLUSION}

In this report we have explained tokenization of assets using Blockchain. We have explained the technologies and the methodology behind it. The upside of this system is the transparency and security throughout the cycle of purchasing tokens to putting up items for tokenization. Unlike the traditional systems Blockchain provides added security. Currently, the designed system is confined to a simple chain but this system can be used as the basis for a more complex tokenization system on Blockchain. We are currently using normal tokens, these tokens can also be replaced by minted tokens. So, it is safe to assume that the market share of cryptocurrency is going to increase and in such times where a deadly virus has spread across the world, we realize the importance of digital payments and currencies and blockchain being a distributed ledger is as safe as it can get, at least with the current technology we possess.

As far as tokenization of assets goes, it is estimated that the 'Era of Tokenization' is approaching and this market is expected to have a market of around $\$ 27$ Trillion by 2027.

\section{REFERENCES}

[1] Nakamoto, Satoshi (2009), "Bitcoin: A peer-to-peer electroniccash system."

URL https:/ / bitcoin.org/bitcoin.pdf/

Kosba, A.(2015), "The Blockchain Model of Cryptography and Privacy-Preserving Smart Contracts" in IACR working paper 2015

[2] Deloitte(2016), "Deloitte. The tokenization of assets is disrupting the financial industry.Are you ready? 2018

[3] K. Korpela (2016), "Digital business ecosystem transformation--towards cloud integration" in 49th Hawaii International Conference on System Sciences (HICSS) 2016

[4] Deloitte,Inside Issue Magzine-19, 2018

[5] Kelsey Taylor, HiTechNectar

[6] Castillo, Michael(2017), "Walmart, Kroger \& Nestle Team with IBM Blockchain to Fight Food Poisoning" in https://www.coindesk.com/walmartkroger -nestle-team-with-ibm-blockchain-to-fightfood-poisoning

[7] Nitin Gaur (2017), "Guidelines for Blockchain adoption in the enterprise: How to compare frameworks" at IBM Blockchain Dev center 